\title{
Solving the preoperative breast MRI conundrum: design and protocol of the MIPA study
}

\author{
Francesco Sardanelli ${ }^{1,2}$ • Rubina M. Trimboli ${ }^{1}$ - Nehmat Houssami ${ }^{3}$ • Fiona J. Gilbert ${ }^{4}$. Thomas H. Helbich ${ }^{5}$. \\ Marina Alvarez Benito ${ }^{6}$. Corinne Balleyguier ${ }^{7}$. Massimo Bazzocchi ${ }^{8}$ • Peter Bult ${ }^{9}$ - Massimo Calabrese ${ }^{10}$. \\ Julia Camps Herrero ${ }^{11,12}$. Francesco Cartia ${ }^{13}$. Enrico Cassano ${ }^{14}$. Paola Clauser ${ }^{5}$. Danubia A. de Andrade ${ }^{15}$. $^{2}$ \\ Marcos F. de Lima Docema ${ }^{16}$. Catherine Depretto ${ }^{13}$. Gabor Forrai ${ }^{17,18}$ • Rossano Girometti ${ }^{8}$. Steven E. Harms ${ }^{19}$. \\ Sarah Hilborne ${ }^{4}$ - Raffaele lenzi ${ }^{20}$ - Marc B. I. Lobbes ${ }^{21,22}$ • Claudio Losio ${ }^{23}$ - Ritse M. Mann ${ }^{24,25}$. \\ Stefania Montemezzi ${ }^{26}$. Inge-Marie Obdeijn ${ }^{27}$. Umit Aksoy Ozcan ${ }^{28}$ • Federica Pediconi ${ }^{29} \cdot$ Heike Preibsch $^{30}$. \\ José L. Raya-Povedano ${ }^{31}$ - Daniela Sacchetto ${ }^{32,33,34}$ • Gianfranco P. Scaperrotta ${ }^{13}$ - Margrethe Schlooz ${ }^{35}$. \\ Botond K. Szabo ${ }^{36}$. Ozden S. Ulus ${ }^{28}$ • Donna B. Taylor ${ }^{37,38}$ • Mireille Van Goethem ${ }^{39}$. Jeroen Veltman ${ }^{40}$. \\ Stefanie Weigel $^{41} \cdot$ Evelyn Wenkel $^{42} \cdot$ Chiara Zuiani $^{8} \cdot$ Giovanni Di Leo $^{2}$
}

Received: 27 December 2019 / Revised: 11 February 2020 / Accepted: 19 March 2020

(C) European Society of Radiology 2020

\begin{abstract}
Despite its high diagnostic performance, the use of breast MRI in the preoperative setting is controversial. It has the potential for personalized surgical management in breast cancer patients, but two of three randomized controlled trials did not show results in favor of its introduction for assessing the disease extent before surgery. Meta-analyses showed a higher mastectomy rate in women undergoing preoperative MRI compared to those who do not. Nevertheless, preoperative breast MRI is increasingly used and a survey from the American Society of Breast Surgeons showed that $41 \%$ of respondents ask for it in daily practice. In this context, a large-scale observational multicenter international prospective analysis (MIPA study) was proposed under the guidance of the European Network for the Assessment of Imaging in Medicine (EuroAIM). The aims were (1) to prospectively and systematically collect data on consecutive women with a newly diagnosed breast cancer, not candidates for neoadjuvant therapy, who are offered or not offered breast MRI before surgery according to local practice; (2) to compare these two groups in terms of surgical and clinical endpoints, adjusting for covariates. The underlying hypotheses are that MRI does not cause additional mastectomies compared to conventional imaging, while reducing the reoperation rate in all or in subgroups of patients. Ninety-six centers applied to a web-based call; 36 were initially selected based on volume and quality standards; 27 were active for enrollment. On November 2018, the target of 7000 enrolled patients was reached. The MIPA study is presently at the analytic phase.

Key Points

- Breast MRI has a high diagnostic performance but its utility in the preoperative setting is controversial.

- A large-scale observational multicenter prospective study was launched to compare women receiving with those not receiving preoperative MRI.

- Twenty-seven centers enrolled more than 7000 patients. The study is presently at the analytic phase.
\end{abstract}

Keywords Breast neoplasms $\cdot$ Breast-conserving surgery $\cdot$ Magnetic resonance imaging $\cdot$ Mastectomy $\cdot$ Prospective studies

Electronic supplementary material The online version of this article (https://doi.org/10.1007/s00330-020-06824-7) contains supplementary material, which is available to authorized users.

Francesco Sardanelli

francesco.sardanelli@unimi.it

Extended author information available on the last page of the article

\section{Abbreviations}

BCS Breast-conserving surgery

EuroAIM European Network for the Assessment of Imaging in Medicine

EUSOBI European Society of Breast Imaging

MRI Magnetic resonance imaging

OR Odds ratio

RCT Randomized controlled trial 


\section{Introduction}

Breast-conserving surgery (BCS) with whole breast irradiation is the best option for operable breast cancers, comparable to mastectomy in terms of survival $[1,2]$, with generally better psychosocial outcomes. However, after BCS, the risk of locoregional recurrences or new ipsilateral/contralateral cancers is 1.0 to $1.5 \%$ per year for 15-20 years [3]. In addition, the rate of positive/close margins after BCS is $26-30 \%[4,5]$ usually leading to reoperation.

The role of preoperative contrast-enhanced breast MRI emerged in this surgical context. It showed an unparalleled sensitivity in comparison with mammography and/or ultrasound [6-12]. When compared to double-reading mammography in 99 mastectomies [13], its sensitivity (81\%) was significantly higher than that of mammography $(66 \%)$. A large study [14] reported over $95 \%$ sensitivity and specificity while another study [15] reported that $23 \%$ of MRI-detected additional tumors were larger and 5\% more biologically important than the index cancer. In meta-analyses, the frequency of MRI-detected additional cancers was around 20\% [16], in agreement with the high rate of multifocal/multicentric cancers found at pathology of mastectomy specimens [17], while the rate of additional contralateral cancers was 4-5\% [16, 18].

Breast MRI has been advocated to personalize the surgical management, reducing reoperation rate and early-detecting contralateral cancers, with a potential for improving diseasefree survival. However, two of three randomized controlled trials (RCT) $[19,20]$ did not confirm better surgical outcomes, and the third [21], focusing on relatively younger women was in favor of MRI. Furthermore, a recent RCT of 360 patients with ductal carcinoma in situ failed to show a significant reduction of the reoperation rate in the MRI group over the control group (20\% versus 27\%) highlighting the need for large sample sizes to examine benefits in subgroups [22].

A meta-analysis [23] found a first-line mastectomy rate significantly higher for the MRI group (16\%) than for the no-MRI group $(8 \%)$, a similar reoperation rate $(12 \%$ versus $11 \%$, respectively), and an overall (first-line plus secondary) mastectomy rate significantly higher for the MRI group (26\% versus $18 \%$, respectively). In patients with invasive lobular histology, the first-line mastectomy rate was $31 \%$ versus $25 \%$, the reoperation rate $11 \%$ versus $18 \%$, and the overall mastectomy rate $43 \%$ versus $40 \%$, respectively (with significance borderline or dependent on adjustments).

An individual patient data meta-analysis [24] showed that the 8-year local and distant recurrence-free survival did not significantly differ between patients locally staged with or without MRI. Another meta-analysis [25] did not find evidence that MRI improved surgical outcomes while the odds for ipsilateral mastectomy (odds ratio [OR] 1.39) and contralateral prophylactic mastectomy (OR 1.91) were significantly increased. In the subgroup of invasive lobular histology, MRI was not associated with an increase in mastectomy rate (OR 1.0 ) but with a reduced probability of reoperation (OR 0.65$)$, although not at statistically significant levels.

Data from these studies are summarized in Table 1.

The discussion regarding the utility of preoperative MRI has not ended yet, with arguments in favor [26, 27] and against [28-30], and remains an unsolved aspect of breast cancer management $[31,32]$. While MRI certainly improves disease extent definition, there is conflicting evidence and uncertainty on whether and in whom it is beneficial, underscoring the need for additional evidence.

The American Society of Breast Surgeons [33] suggests "Don't routinely order breast MRI in new breast cancer patients." However, regardless of evidence and recommendations, a survey from that society [34] showed that $41 \%$ of responding surgeons use preoperative MRI in daily practice. Another survey [35] showed a surgeons' propensity for requesting MRI in case of (in decreasing order) BRCA mutations; familial/personal breast cancer history; dense breasts; age $<40$; axillary nodal involvement; mammographically occult tumor; multifocal/multicentric disease at conventional imaging; invasive lobular histology; triple-negative cancer; T2-T3 stage; candidates for mastectomy requesting breast-conserving surgery (BCS); and radiologist's discretion.

\section{A prospective multicenter trial to solve the conundrum}

An international group (European Network for the Assessment of Imaging in Medicine, EuroAIM; https:// www.eibir.org/initiatives/euroaim/) established a collaborative plan to conduct a large-scale observational multicenter international prospective analysis (MIPA) study to be performed in institutions providing high-volume breast MRI and high-quality standards. The aims were (1) to prospectively and systematically collect data on consecutive women with a newly diagnosed first breast cancer, who are not candidates to neoadjuvant therapy, and who are offered MRI (MRI group) or not (no-MRI group) as part of local breast cancer management; (2) to compare these two groups in terms of characteristics, and surgical and clinical endpoints. The underlying hypotheses are that MRI does not cause additional mastectomies compared to conventional imaging, while reducing the reoperation rate in all or subgroups of patients.

\section{International open call}

The MIPA study was undertaken under the initiative and responsibility of the EuroAIM and endorsed by the European Society of Breast Imaging (EUSOBI). Bayer Healthcare provided an unconditional research grant for the conduct of the study. 


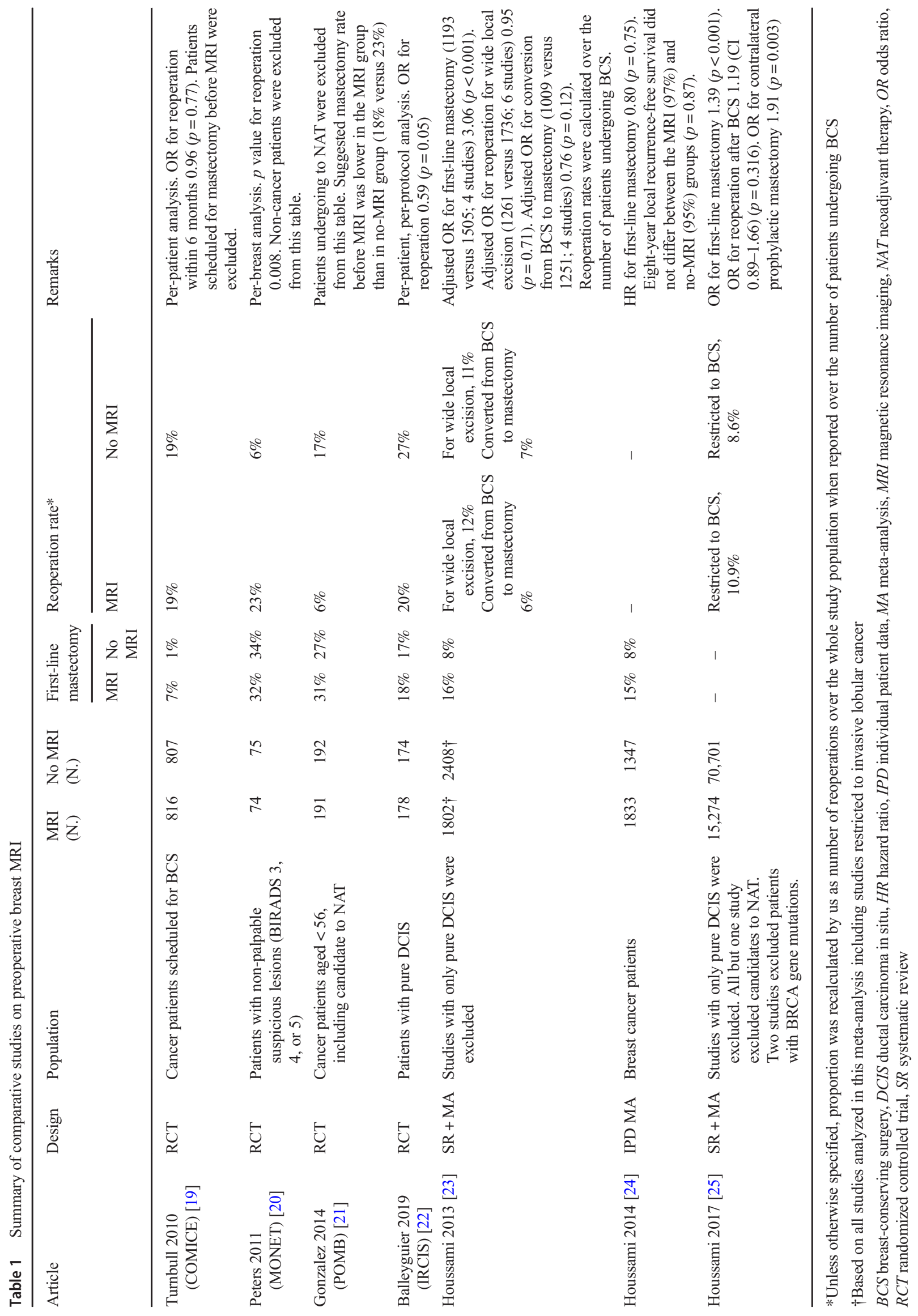


In mid-2012, an open call was published on the EuroAIM website and circulated within the EUSOBI members. Applying centers had to confirm a series of preliminary conditions pertaining to quality of MRI, focusing on equipment and workload (Table 1 of Supplemental material). Moreover, they had to anticipate (1) the approximate number of patients potentially recruitable; and (2) the local rate of MRI performed in women newly diagnosed with breast cancer.

Once selected, centers provided technical details of the MRI protocols applied locally. The coordinating center approved only MRI protocols following technical recommendations issued by international societies such us the European Society of Breast Cancer Specialists (EUSOMA) [36], the EUSOBI [37], and the American College of Radiology [38]. Details are shown in Table 2.

\section{Study design and population}

MIPA is a multicenter observational prospective study enrolling women newly diagnosed with breast cancer. No experimental intervention was planned. Centers were free to perform or not perform MRI according to local practice. Thus, two concurrent groups resulted ex post by whether women underwent preoperative staging using conventional imaging only (no-MRI group) or also received contrast-enhanced MRI (MRI group) in addition to conventional imaging, representing real-world breast cancer management (study registration ISRCTN41143178).

The study was conducted in accordance with the Declaration of Helsinki and was firstly approved on January 29, 2013, by the ethics committee at the coordinating center (protocol number 2784). Thereafter, ethical approval was

Table 2 Preoperative breast MRI: technical requirements and imaging protocols of the MIPA study

$\begin{array}{ll}\text { Technical requirements } & \\ \text { Magnetic field strength } & \geq 1.5 \mathrm{~T} \\ \text { Gradients power } & \geq 4 \\ \text { Channels of dedicated coil } & \\ \text { Technical protocol } & \\ \text { One of the following T2-weighted sequences (axial or sagittal): } \\ \text { - Fast/turbo spin-echo } \pm \text { fat-sat } \\ \text { - Short tau inversion recovery (STIR) } \\ \text { - Spectral pre-saturation with inversion recovery (SPIR) } \\ \text { Contrast agent type and dose: extracellular gadolinium-based contrast } \\ \text { agent, } 0.1 \text { mmol/kg } \\ \text { Dynamic study (2D or 3D T1-weighted gradient-echo sequence } \pm \\ \text { fat-saturation, axial or sagittal) } \\ \text { - Slice thickness } \leq 3 \mathrm{~mm} \\ \text { - In-plane resolution } \leq 1.5 \mathrm{~mm}^{2} \text { (preferably } \leq 1 \mathrm{~mm}^{2} \text { ) } \\ \text { - Temporal resolution } \leq 120 \mathrm{~s}\end{array}$

$2 D$ two-dimensional, $3 D$ three-dimensional obtained at each center. All participants signed an informed consent allowing the use of their data according the observational design.

The coordinating center at the Research Hospital (IRCCS) Policlinico San Donato (San Donato Milanese, Milan, Italy) was charged to ensure that the research data were accurate, complete, and consistent. The description of the database set up is reported in the supplemental material.

The study population included women aged 18 to 80 years newly diagnosed with a first breast cancer amenable to upfront surgery. Exclusion criteria included indication to neoadjuvant therapy, pregnancy, personal history of invasive or ductal in situ breast cancer, personal history of any cancer, evidence of distant metastases at enrollment, and inability to provide informed consent. Women with contraindications to MRI or to gadolinium-based contrast agents were included in the noMRI group. The study flowchart is shown in Fig. 1.

\section{Study endpoints and statistical analysis}

Primary surgical endpoints for the two groups are (1) the rate of mastectomies actually performed; and (2) the reoperation rate for close or positive margins among patients undergoing BCS. A secondary surgical endpoint for the MRI group only is the rate of change, after MRI, to more extended unilateral BCS (wider excision or multiple excisions) or to less extended $\mathrm{BCS}$, or from unilateral BCS to bilateral BCS or vice versa. Secondary clinical endpoints for the two groups are the rate of (1) ipsilateral recurrences, (2) contralateral breast cancers, and (3) distant metastases at 5-year follow-up.

Assuming a reoperation rate due to positive/close margins in the no-MRI group of $20 \%$, we anticipated a reoperation rate in the MRI group of $15 \%$. To detect this reduction with an $\alpha$ error of 0.05 and $90 \%$ statistical power, 1250 women in each group (total 2500 women) should have been enrolled. To allow for $10 \%$ losses/missing data during follow-up, $1400 \times 2=$ 2800 women would have been needed. As the primary endpoint (reoperation rate) will be compared also in the subgroup of patients with ductal carcinoma in situ, which represents about $20-25 \%$ of all breast cancers $[39,40]$, the target sample size was set at 7000 patients to support subgroup analyses. Considering the large dataset, the sample size will allow explorative analyses for further hypotheses including other histology subgroups (e.g., lobular).

The two concurrent groups will be initially compared in terms of baseline characteristics such as demographics and breast density. As both groups receive conventional imaging, baseline characteristics include the surgical plan based on conventional imaging only, as defined by the multidisciplinary team or through direct interaction between radiologists and surgeons. As this is an observational study, this comparison can provide an insight on criteria used by physicians when ordering preoperative breast MRI that might cause selection 


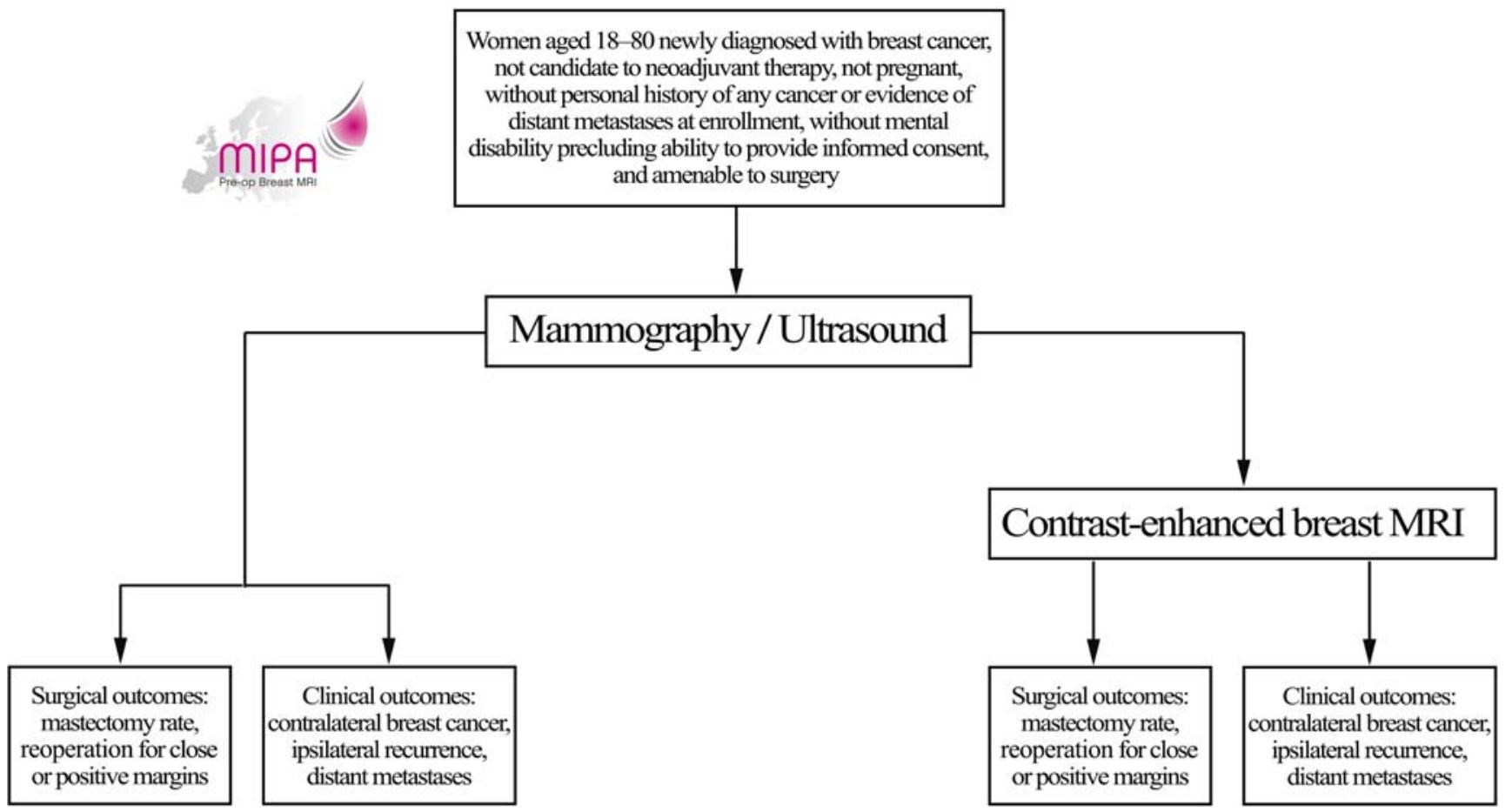

Fig. 1 Flowchart of the prospective observational multicentre MIPA study. Allocation to MRI was based on local clinical practice

biases. To this aim, the odds ratio (OR) of plan for mastectomy after conventional imaging will be calculated for the MRI group relative to the no-MRI group.

Depending on distributions, comparisons of continuous variables will be carried out using Student $t$ test or Mann-Whitney $U$ test for independent data, and using $\chi^{2}$ test for categorical variables. Variables that will be shown to be significantly different between the two groups will be considered as covariates when the two groups will be compared in analyses.

For the primary endpoints, statistical analysis will be performed on a per-patient basis. The two concurrent groups will be compared by calculating the OR for mastectomy for the MRI group over the no-MRI group, raw and adjusted for covariates. A similar analysis will be carried out for the reoperation rate in patients undergoing BCS. Moreover, as in a before-after intra-individual study design, the rate of mastectomy before and after MRI in the MRI group will be compared using the McNemar test for paired data. Hence, changes of treatment planning from BCS based on conventional imaging as well as on available clinical examination and pathological data to mastectomy after MRI may compensate for the opposite change.

For secondary surgical endpoints regarding the MRI group only, per-breast distributions will be calculated using the same methodology described above for primary endpoints. Conversely, secondary clinical endpoints will be determined on a per-patient basis. Further analyses will be performed, including investigation of homogeneity across centers and temporal trends for both primary and secondary endpoints.

\section{Timeline}

In about 2 months following the open call, a total of 96 centers applied from all over the world. Thirty-six centers were initially selected based on pre-defined criteria: (1) the full respect of quality standards requested by the call (see Table 1 of Supplemental material); (2) the anticipated total number of patients that could potentially be enrolled in the center; and (3) the anticipated rate of patients that usually undergo preoperative MRI at the center. This allowed careful selection of centers among those with the highest volumes of breast MRI whilst also ensuring to balance the expected rate of MRI at about $50 \%$.

After the study had begun, nine centers abandoned the study or never enrolled patients, four centers started the enrollment with very few patients but did not provide valid data; four centers were added later to replace the vacancies, so 27 centers were actively enrolling. The list of the 27 active centers is shown in Table 3.

During the pilot procedure run, few concerns were raised from local investigators and were fixed promptly. Valuable input came from centers for a further refinement of the electronic case report form, in particular, due to wording that was potentially misleading. The first woman was enrolled in June 2013. On November 30, 2018, the target of 7000 enrolled breast cancer patients was reached. The distribution of patients enrolled in each participating center is shown in Table 3. The enrolment is ended, while the follow-up should end by November 2023. Data cleaning and statistical analysis 
Table 3 List of the 27 centers participating in the MIPA study and number of enrolled patients

Center

Enrolled patients

Radboud University Medical Center, Nijmegen, The Netherlands 956

Azienda Ospedaliero-Universitaria Santa Maria della Misericordia, Udine, Italy 619

Fondazione IRCCS Istituto Nazionale dei Tumori, Milan, Italy 504

University of Cambridge, Addenbrooke's Hospital, Cambridge, UK 485

Hospital Universitario Reina Sofia, Cordoba, Spain $\quad 439$

Acibadem Mehmet Ali Aydinlar University School of Medicine, Istanbul, Turkey $\quad 400$

Medical University of Vienna, Vienna, Austria 368

Sociedade Beneficente De Senhoras, Hospital Sirio Libanes, Sao Paulo, Brazil 301

Maastricht University Medical Center, Maastricht, The Netherlands 265

IEO, Istituto Europeo di Oncologia, Milan, Italy 248

Radiologic Institute University, Erlangen, Germany 246

University Hospital, Tübingen, Germany 244

IRCCS Azienda Ospedaliera Universitaria San Martino, Istituto Nazionale per la Ricerca sul 233

Cancro, Genoa, Italy

Antwerp University Hospital, Antwerp, Belgium 228

Maatschap Radiologie Oost-Nederland, Oldenzaal, The Netherlands 207

Institut de cancérologie Gustave-Roussy 195

Sapienza University, Policlinico Umberto I, Rome, Italy 191

Erasmus Medical Center, University Hospital Rotterdam, Rotterdam, The Netherlands $\quad 178$

Azienda Ospedaliera Universitaria Integrata Verona, Verona, Italy 166

The Breast Center of Northwest Arkansas, Fayetteville, USA 142

Central Military Hospital, Budapest, Hungary 132

IRCCS Ospedale San Raffaele, Milan, Italy $\quad 113$

Hospital de la Ribera, Alzira, Spain $\quad 95$

Royal Perth Hospital, Perth, Australia $\quad 66$

University Hospital, Münster, Germany $\quad 66$

Azienda Ospedaliera Universitaria Policlinico Paolo Giaccone, Palermo, Italy $\quad 40$

BHR Hospitals NHS Trust, Romford, UK 27

$\begin{array}{ll}\text { Total } & 7154\end{array}$

The study was coordinated by the Research Hospital (IRCCS) Policlinico San Donato, San Donato Milanese, Milan, Italy

of the baseline data is being conducted and the first paper with the main results will be submitted by 2020 .

\section{Conclusion}

The use of preoperative MRI of the breast is controversial, mainly because it can affect the surgical planning and yet the evidence on its effect and clinical benefit has been discordant. The MRI diagnosis of additional cancers can change a BCS into mastectomy also when those lesions would be successfully treated by whole breast irradiation and/or systemic therapy, working against reducing surgical aggressiveness.

In the first years of breast MRI, the variable reported specificity [41, 42] combined with a limited use of target ultrasound and the lack of MRI-guidance for biopsy/localization also made MRI false-positives difficult to manage [19]. To transfer knowledge about disease extent demonstrated by MRI, performed in the prone position, to the surgical theater with the patient in supine position, is not easy [43]. A learning curve gradually improved the performance of radiologists in using targeted ultrasound [44], second-look mammography/ tomosynthesis [45], and MRI-guidance [46]. Surgeons as well needed time to learn how to exploit the additional information from preoperative breast MRI.

The application of evidence-based medicine [47] is difficult in this setting. Preoperative MRI joins three clinical issues: (1) measuring the size of the index lesion; (2) searching for ipsilateral additional cancers; and (3) screening for contralateral cancers. While the first requires cohort studies correlating sizes at MRI and histopathology, the third is a screeninglike task, theoretically needing RCTs before implementation $[47,48]$; the second issue is arguably in the middle. Considering the treatments still prevalent for breast cancer 
including whole breast irradiation following BCS, mastectomy for multicentric cancers, and variable systemic therapy, effects of preoperative MRI are difficult to assess.

The strength of the MIPA study is its special design. In fact, two designs were combined to derive the highest evidence possible: a case-control approach allowing the comparison of women undergoing MRI and women not undergoing MRI, and a before-after approach to have a direct measure of the MRI impact on surgery. The well-known limitations of the case-control approach will, in turn, result in an advantage for the MIPA study. In fact, whatever the difference between the two groups in terms of mastectomy/reoperation rate, the knowledge of the treatment plan after conventional imaging even in the MRI group will provide evidence on possible selection bias. From the other side, the before-after approach will allow not only the estimation of the additional mastectomy rate independently prompted by MRI (that may also be negative, meaning that MRI reduces the mastectomy rate) but also the evaluation of the changes of surgical extension in those women undergoing BCS, from less extensive to more extensive and vice versa.

Although the lack of randomization is a limitation of the MIPA study, it can be considered an effective and pragmatic way to provide evidence that is closer to the real-world approach $[49,50]$. Especially from the intra-individual part of the study (comparing the plan for mastectomy before and after MRI), we will provide a clear insight into MRI's impact on patients' surgical management. Moreover, thanks to large number of patients and data that were collected, we will be able to answer several other questions. In particular, subgroup analyses will ascertain which patients will benefit the most from preoperative MRI. Correlation analyses will instead clarify the role of potential predictors, while follow-up data will provide evidence on cancer recurrences.

In conclusion, a large-scale real-world prospective multicenter study investigating the role and effect of MRI in the preoperative setting was launched internationally. This exemplar of the joint efforts of 27 centers enabled the target of 7000 women with newly diagnosed breast cancer to be reached and the study is presently at the analytic phase.

Acknowledgments The study was promoted by the European Network for the Assessment of Imaging in Medicine (EuroAIM), initiative of the European Institute for Biomedical Imaging Research (EIBIR) and endorsed by the European Society of Breast Imaging. The authors thank Bayer Healthcare that provided an unconditional research grant, in particular Dr. Stephanie Schermuck-Joschko (who passed away for a car accident after the study started) and Dr. Jan Endrikat. The authors also thank Monika Hierath, Eva Haas, Katharina Krischak, and Peter Gordebeke from the EIBIR staff which managed all the administrative work of this study.

The following persons collaborated at individual centers: Lucia Camera, MD, Department of Radiology, Azienda Ospedaliera Universitaria Integrata, Verona, Italy; Sara Mirandola, MD, Department of Surgery, Azienda Ospedaliera Universitaria Integrata, Verona, Italy;
Marta Maria Panzeri, MD, Department of Breast Radiology, IRCCS Ospedale San Raffaele, Milan, Italy; Maria A. Rodi Carvalho Barros Bernardes, MD and Vera L. Nunes Aguillar, MD, PhD, Department of Radiology, Hospital Sirio Libanes, Sao Paulo, Brazil; Katja SiegmannLuz, MD and Benjamin Wiesinger, MD, Department of Diagnostic and Interventional Radiology, University Hospital of Tuebingen, Germany; James M. Anderson, Max Hobbs, and Wanda Gunawan, Royal Perth Hospital, Perth, Australia.

Funding information This study has received funding by Bayer Healthcare.

\section{Compliance with ethical standards}

Guarantor The scientific guarantor of this publication is Professor Francesco Sardanelli (Università degli Studi di Milano).

Conflict of interest The authors of this manuscript declare the following relationships with companies:

Thomas Helbich receives research funding from Siemens, Guerbet, Bracco, Hologic and Nocomed.

Francesco Sardanelli received research grants from and is a member of the speakers' bureau of GE Healthcare, Bayer, and Bracco; he is also a member of the Bracco Advisory Group.

Fiona J Gilbert received research grants from GE Healthcare, GSK, and Hologic and had research collaborations with Volpara and Bayer. She is an NIHR senior investigator and receives funding from the Cambridge BRC.

Marc Lobbes received research grant and is a member of the speakers' bureau of GE Healthcare.

Nehmat Houssami receives research funding via a National Breast Cancer Foundation (NBCF Australia) Breast Cancer Research Leadership Fellowship.

Statistics and biometry One of the authors has significant statistical expertise.

Informed consent Written informed consent was obtained from all subjects (patients) in this study.

Ethical approval Institutional Review Board approval was obtained.
Methodology
- prospective
- observational
- multicenter study

\section{References}

1. Veronesi U, Cascinelli N, Mariani L et al (2002) Twenty-year follow-up of a randomized study comparing breast-conserving surgery with radical mastectomy for early breast cancer. N Engl J Med 347: 1227-1232. https://doi.org/10.1056/NEJMoa020989

2. Fisher B, Anderson S, Bryant J et al (2002) Twenty-year follow-up of a randomized trial comparing total mastectomy, lumpectomy, and lumpectomy plus irradiation for the treatment of invasive breast cancer. N Engl J Med 347:1233-1241. https://doi.org/10.1056/ NEJMoa022152

3. Bucchi L, Belli P, Benelli E et al (2016) Recommendations for breast imaging follow-up of women with a previous history of breast cancer: position paper from the Italian Group for 
Mammography Screening (GISMa) and the Italian College of Breast Radiologists (ICBR) by SIRM. Radiol Med 121:891-896. https://doi.org/10.1007/s11547-016-0676-8

4. Houssami N, Macaskill P, Marinovich ML et al (2010) Metaanalysis of the impact of surgical margins on local recurrence in women with early-stage invasive breast cancer treated with breastconserving therapy. Eur J Cancer 46:3219-3232. https://doi.org/10. 1016/j.ejca.2010.07.043

5. Kurniawan ED, Wong MH, Windle I et al (2008) Predictors of surgical margin status in breast-conserving surgery within a breast screening program. Ann Surg Oncol 15:2542-2549. https://doi.org/ 10.1245/s10434-008-0054-4

6. Kuhl C, Weigel S, Schrading S et al (2010) Prospective multicenter cohort study to refine management recommendations for women at elevated familial risk of breast cancer: the EVA trial. J Clin Oncol 28:1450-1457. https://doi.org/10.1200/JCO.2009.23.0839

7. Sardanelli F, Podo F, Santoro F et al (2011) Multicenter surveillance of women at high genetic breast cancer risk using mammography, ultrasonography, and contrast-enhanced magnetic resonance imaging (the high breast cancer risk Italian 1 study): final results. Invest Radiol 46:94-105. https://doi.org/10.1097/RLI.0b013e3181f3fcdf

8. Obdeijn IM, Winter-Warnars GA, Mann RM et al (2014) Should we screen BRCA1 mutation carriers only with MRI? A multicenter study. Breast Cancer Res Treat 144:577-582. https://doi.org/10. 1007/s10549-014-2888-8

9. Chiarelli AM, Prummel MV, Muradali D et al (2014) Effectiveness of screening with annual magnetic resonance imaging and mammography: results of the initial screen from the Ontario high risk breast screening program. J Clin Oncol 32:2224-2230. https://doi. org $/ 10.1200 / \mathrm{JCO} .2013 .52 .8331$

10. Riedl CC, Luft N, Bernhart C et al (2015) Triple-modality screening trial for familial breast cancer underlines the importance of magnetic resonance imaging and questions the role of mammography and ultrasound regardless of patient mutation status, age, and breast density. J Clin Oncol 33:1128-1135. https://doi.org/10.1200/JCO. 2014.56.8626

11. Phi XA, Saadatmand S, De Bock GH et al (2016) Contribution of mammography to MRI screening in BRCA mutation carriers by BRCA status and age: individual patient data meta-analysis. Br J Cancer 114:631-637. https://doi.org/10.1038/bjc.2016.32

12. Lo G, Scaranelo AM, Aboras H et al (2017) Evaluation of the utility of screening mammography for high-risk women undergoing screening breast MR imaging. Radiology 285:36-43. https://doi. org/10.1148/radiol.2017161103

13. Sardanelli F, Giuseppetti GM, Panizza P et al (2004) Sensitivity of MRI versus mammography for detecting foci of multifocal, multicentric breast cancer in fatty and dense breasts using the whole-breast pathologic examination as a gold standard. AJR Am J Roentgenol 183:1149-1157. https://doi.org/10.2214/ajr.183.4. 1831149

14. Sardanelli F, Newstead GM, Putz B et al (2016) Gadobutrolenhanced magnetic resonance imaging of the breast in the preoperative setting: results of 2 prospective international multicenter phase III studies. Invest Radiol 51:454-461. https://doi.org/10. 1097/RLI.0000000000000254

15. Iacconi C, Galman L, Zheng J et al (2016) Multicentric cancer detected at breast MR imaging and not at mammography: important or not? Radiology 279:378-384. https://doi.org/10.1148/radiol. 2015150796

16. Plana MN, Carreira C, Muriel A et al (2012) Magnetic resonance imaging in the preoperative assessment of patients with primary breast cancer: systematic review of diagnostic accuracy and metaanalysis. Eur Radiol 22:26-38. https://doi.org/10.1007/s00330011-2238-8

17. Holland R, Veling SH, Mravunac M, Hendriks JH (1985) Histologic multifocality of Tis, T1-2 breast carcinomas.
Implications for clinical trials of breast-conserving surgery. Cancer 56:979-990. https://doi.org/10.1002/10970142(19850901)56:5<979::aid-cncr2820560502>3.0.co;2-n

18. Brennan ME, Houssami N, Lord S et al (2009) Magnetic resonance imaging screening of the contralateral breast in women with newly diagnosed breast cancer: systematic review and meta-analysis of incremental cancer detection and impact on surgical management. J Clin Oncol 27:5640-5649. https://doi.org/10.1200/JCO.2008.21. 5756

19. Turnbull L, Brown S, Harvey I et al (2010) Comparative effectiveness of MRI in breast cancer (COMICE) trial: a randomized controlled trial. Lancet 375:563-571. https://doi.org/10.1016/S01406736(09)62070-5

20. Peters NH, van Esser S, van den Bosch MA et al (2011) Preoperative MRI and surgical management in patients with nonpalpable breast cancer: the MONET - randomised controlled trial. Eur J Cancer 47:879-886. https://doi.org/10.1016/j.ejca. 2010.11.035

21. Gonzalez V, Sandelin K, Karlsson A et al (2014) Preoperative MRI of the breast $(\mathrm{POMB})$ influences primary treatment in breast cancer: a prospective, randomized, multicenter study. World J Surg 38: 1685-1693. https://doi.org/10.1007/s00268-014-2605-0

22. Balleyguier C, Dunant A, Ceugnart L et al (2019) Preoperative breast magnetic resonance imaging in women with local ductal carcinoma in situ to optimize surgical outcomes: results from the randomized phase III trial IRCIS. J Clin Oncol 37:885-892. https:// doi.org/10.1200/JCO.18.00595

23. Houssami N, Turner R, Morrow M (2013) Preoperative magnetic resonance imaging in breast cancer: meta-analysis of surgical outcomes. Ann Surg 257:249-255. https://doi.org/10.1097/SLA. 0b013e31827a $8 \mathrm{~d} 17$

24. Houssami N, Turner R, Macaskill P et al (2014) An individual person data meta-analysis of preoperative magnetic resonance imaging and breast cancer recurrence. J Clin Oncol 32:392-401. https://doi.org/10.1200/JCO.2013.52.7515

25. Houssami N, Turner RM, Morrow M (2017) Meta-analysis of preoperative magnetic resonance imaging (MRI) and surgical treatment for breast cancer. Breast Cancer Res Treat 165:273-283. https://doi.org/10.1007/s10549-017-4324-3

26. Sardanelli F (2010) Additional findings at preoperative MRI: a simple golden rule for a complex problem? Breast Cancer Res Treat 124:717-721. https://doi.org/10.1007/s10549-010-1144-0

27. Sardanelli F, Trimboli RM (2012) Preoperative MRI: did randomized trials conclude the debate? Eur J Radiol 81(Suppl 1):S135S136. https://doi.org/10.1016/S0720-048X(12)70056-6

28. Solin LJ (2010) Counterview: pre-operative breast MRI (magnetic resonance imaging) is not recommended for all patients with newly diagnosed breast cancer. Breast 19:7-9. https://doi.org/10.1016/j. breast.2009.11.004

29. Houssami N, Solin LJ (2010) An appraisal of pre-operative MRI in breast cancer: more effective staging of the breast or much ado about nothing? Maturitas 67:291-293. https://doi.org/10.1016/j. maturitas.2010.08.008

30. Jatoi I, Benson JR (2013) The case against routine preoperative breast MRI. Future Oncol 9:347-353. https://doi.org/10.2217/fon. 12.186

31. Gupta D, Billadello L (2017) Breast MR imaging in newly diagnosed breast cancer. Radiol Clin North Am 55:541-552. https://doi. org/10.1016/j.rcl.2016.12.008

32. Ray KM, Hayward JH, Joe BN (2018) Role of MR imaging for the locoregional staging of breast cancer. Magn Reson Imaging Clin N Am 26:191-205. https://doi.org/10.1016/j.mric.2017.12.008

33. American Society of Breast Surgeons web-site. http://www. choosingwisely.org/societies/american-society-of-breastsurgeons/. Accessed 28 Aug 2019 
34. Parker A, Schroen AT, Brenin DR (2013) MRI utilization in newly diagnosed breast cancer: a survey of practicing surgeons. Ann Surg Oncol 20:2600-2606. https://doi.org/10.1245/s10434-013-2934-5

35. Lee J, Tanaka E, Eby PR et al (2017) Preoperative breast MRI: surgeons' patient selection patterns and potential bias in outcomes analyses. AJR Am J Roentgenol 208:923-932. https://doi.org/10. 2214/AJR.16.17038

36. Sardanelli F, Boetes C, Borisch B et al (2010) Magnetic resonance imaging of the breast: recommendations from the EUSOMA working group. Eur J Cancer 46:1296-1316. https://doi.org/10.1016/j. ejca.2010.02.015

37. Mann RM, Balleyguier C, Baltzer PA et al (2015) Breast MRI: EUSOBI recommendations for women's information. Eur Radiol 25:3669-3678. https://doi.org/10.1007/s00330-015-3807-z

38. American College of Radiology (2018) Practice parameter for the performance of contrast-enhanced magnetic resonance imaging (MRI) of the breast. https://www.acr.org/-/media/ACR/Files/ Practice-Parameters/mr-contrast-breast.pdf. Accessed 28 Aug 2019

39. Allegra CJ, Aberle DR, Ganschow P et al (2009) NIH state-of-thescience conference statement: diagnosis and management of ductal carcinoma in situ (DCIS). NIH Consens State Sci Statements 26:127 https://consensus.nih.gov/2009/dcisstatement.htm

40. Bleyer A, Welch HG (2012) Effect of three decades of screening mammography on breast-cancer incidence. N Engl J Med 367: 1998-2005. https://doi.org/10.1056/NEJMoa1206809

41. Harms SE, Flamig DP, Hesley KL et al (1993) MR imaging of the breast with rotating delivery of excitation off resonance: clinical experience with pathologic correlation. Radiology 187:493-501. https://doi.org/10.1148/radiology.187.2.8475297

42. Kaiser WA (1994) False-positive results in dynamic MR mammography. Causes, frequency, and methods to avoid. Magn Reson Imaging Clin N Am 2:539-555

43. Carbonaro LA, Tannaphai P, Trimboli RM, Verardi N, Fedeli MP, Sardanelli F (2012) Contrast-enhanced breast MRI: spatial displacement from prone to supine patient's position. Preliminary results. Eur J Radiol 81:e771-e774. https://doi.org/10.1016/j.ejrad. 2012.02.013

44. Spick C, Baltzer PA (2014) Diagnostic utility of second-look US for breast lesions identified at MR imaging: systematic review and meta-analysis. Radiology 273:401-409. https://doi.org/10.1148/ radiol.14140474

45. Clauser P, Carbonaro LA, Pancot M et al (2015) Additional findings at preoperative breast MRI: the value of second-look digital breast tomosynthesis. Eur Radiol 25:2830-2839. https://doi.org/10.1007/ s00330-015-3720-5

46. Spick C, Schernthaner M, Pinker K et al (2016) MR-guided vacuum-assisted breast biopsy of MRI-only lesions: a single center experience. Eur Radiol 26:3908-3916. https://doi.org/10.1007/ s00330-016-4267-9

47. Oxford Centre for Evidence-based Medicine. Levels of Evidence. https://www.cebm.net/2009/06/oxford-centre-evidence-basedmedicine-levels-evidence-march-2009/. Accessed 16 Aug 2019

48. The Council of the European Union (2003) Council recommendation of 2 December 2003 on cancer screening (2003/878/EC). https://ec.europa.eu/jrc/sites/jrcsh/files/2 December 2003cancerscreening.pdf. Accessed 16 Aug 2019

49. Nabhan C, Klink A, Prasad V (2019) Real-world evidence-what does it really mean? JAMA Oncol 5:781-783. https://doi.org/10. 1001/jamaoncol.2019.0450

50. Corrigan-Curay J, Sacks L, Woodcock J (2018) Real-world evidence and real-world data for evaluating drug safety and effectiveness. JAMA 320:867-868. https://doi.org/10.1001/jama.2018. 10136

Publisher's note Springer Nature remains neutral with regard to jurisdictional claims in published maps and institutional affiliations.

\section{Affiliations}

Francesco Sardanelli ${ }^{1,2} \cdot$ Rubina M. Trimboli $^{1} \cdot$ Nehmat Houssami $^{3}$ • Fiona J. Gilbert ${ }^{4}$ - Thomas H. Helbich ${ }^{5}$. Marina Alvarez Benito ${ }^{6}$. Corinne Balleyguier ${ }^{7}$. Massimo Bazzocchi ${ }^{8} \cdot$ Peter Bult $^{9}$. Massimo Calabrese ${ }^{10}$. Julia Camps Herrero ${ }^{11,12}$. Francesco Cartia ${ }^{13}$. Enrico Cassano ${ }^{14}$. Paola Clauser ${ }^{5}$. Danubia A. de Andrade ${ }^{15}$. Marcos F. de Lima Docema ${ }^{16}$. Catherine Depretto ${ }^{13}$. Gabor Forrai ${ }^{17,18} \cdot$ Rossano Girometti $^{8}$. Steven E. Harms ${ }^{19}$. Sarah Hilborne ${ }^{4}$ - Raffaele lenzi ${ }^{20}$ • Marc B. I. Lobbes ${ }^{21,22}$. Claudio Losio ${ }^{23}$ • Ritse M. Mann 24,25 Stefania Montemezzi ${ }^{26} \cdot$ Inge-Marie Obdeijn ${ }^{27}$. Umit Aksoy Ozcan ${ }^{28} \cdot$ Federica Pediconi $^{29} \cdot$ Heike Preibsch $^{30}$. José L. Raya-Povedano ${ }^{31}$ • Daniela Sacchetto ${ }^{32,33,34}$. Gianfranco P. Scaperrotta ${ }^{13}$. Margrethe Schlooz ${ }^{35}$. Botond K. Szabo ${ }^{36}$. Ozden S. Ulus ${ }^{28}$. Donna B. Taylor ${ }^{37,38}$. Mireille Van Goethem ${ }^{39}$. Jeroen Veltman ${ }^{40}$. Stefanie Weigel ${ }^{41} \cdot$ Evelyn Wenkel $^{42} \cdot$ Chiara Zuiani $^{8} \cdot$ Giovanni Di Leo $^{2}$

1 Department of Biomedical Sciences for Health, Università degli Studi di Milano, Via Morandi 30, 20097 San Donato Milanese, Italy

2 Unit of Radiology, IRCCS Policlinico San Donato, Via Morandi 30, 20097 San Donato Milanese, Italy

3 Sydney School of Public Health, Faculty of Medicine and Health, The University of Sydney, Camperdown, NSW, Australia

4 Department of Radiology, Cambridge Biomedical Campus, University of Cambridge, Cambridge, UK
5 Department of Biomedical Imaging and Image-guided Therapy, Division of Molecular and Gender Imaging, Medical University of Vienna, Vienna, Austria

6 Department of Radiology, Hospital Universitario Reina Sofía, Córdoba, Spain

7 Department of Radiology, Gustave-Roussy Institute, Villejuif, France

8 Institute of Radiology, Department of Medicine, University of Udine, University Hospital S. Maria della Misericordia, Udine, Italy 
9 Department of Pathology, Radboud University Medical Center, Nijmegen, The Netherlands

10 Unit of Breast Radiology, IRCCS Ospedale Policlinico San Martino, Genoa, Italy

11 Department of Radiology, University Hospital of La Ribera, Alzira, Spain

12 Jefa Corporativa del Área de la Mama, Ribera Salud Grupo, Valencia, Spain

13 Unit of Breast Imaging, Fondazione IRCCS Istituto Nazionale dei Tumori, Milan, Italy

14 Breast Imaging Division, European Institute of Oncology, Milan, Italy

15 Department of Breast Surgery, Hospital Sírio Libanês, Sao Paulo, Brazil

16 Department of Radiology, Hospital Sírio Libanês, Sao Paulo, Brazil

17 Department of Radiology, MHEK Teaching Hospital University Semmelweis, Budapest, Hungary

18 Department of Radiology, Duna Medical Center, Budapest, Hungary

19 Breast Center of Northwest Arkansas, Fayetteville, AR, USA

20 Department of Radiology Di.Bi.MED, University of Palermo, Policlinico P. Giaccone, Palermo, Italy

21 Department of Radiology and Nuclear Medicine, Maastricht University Medical Center, Maastricht, The Netherlands

22 Department of Radiology, Zuyderland Medical Center, SittardGeleen, The Netherlands

23 Department of Breast Radiology, IRCCS Ospedale San Raffaele, Milan, Italy

24 Department of Radiology and Nuclear Medicine, Radboud University Nijmegen Medical Center, Nijmegen, The Netherlands

25 Department of Radiology, The Netherlands Cancer Institute, Amsterdam, The Netherlands
26 Department of Radiology, University Hospital of Verona, Verona, Italy

27 Department of Radiology and Nuclear Medicine, Erasmus University Medical Center, Rotterdam, The Netherlands

28 Unit of Radiology, Acibadem Mehmet Ali Aydinlar University School of Medicine, Istanbul, Turkey

29 Department of Radiological, Oncological and Pathological Sciences, Sapienza University of Rome, Rome, Italy

30 Department of Diagnostic and Interventional Radiology, University Hospital of Tübingen, Tübingen, Germany

31 Deparment of Radiology, Hospital Universitario Reina Sofía, Córdoba, Spain

32 Kiwifarm Srl, La Morra, Italy

33 Disaster Medicine Service 118, ASL CN1, Saluzzo, Italy

34 CRIMEDIM, Research Center in Emergency and Disaster Medicine, Università del Piemonte Orientale, Novara, Italy

35 Department of Surgery, Radboud University Medical Center, Nijmegen, The Netherlands

36 Department of Radiology, Barking Havering and Redbridge University Hospitals NHS Trust, London, UK

37 Medical School, Faculty of Health and Medical Sciences, University of Western Australia, Perth, Australia

38 Department of Radiology, Royal Perth Hospital, Perth, Australia

39 Gynecological Oncology Unit, Department of Obstetrics and Gynecology, Department of Radiology, Multidisciplinary Breast Clinic, Antwerp University Hospital-University of Antwerp, Antwerp, Belgium

40 Maatschap Radiologie Oost-Nederland, Oldenzaal, The Netherlands

41 Institute of Clinical Radiology and Reference Center for Mammography, University of Münster, Münster, Germany

42 Department of Radiology, University Hospital of Erlangen, Erlangen, Germany 